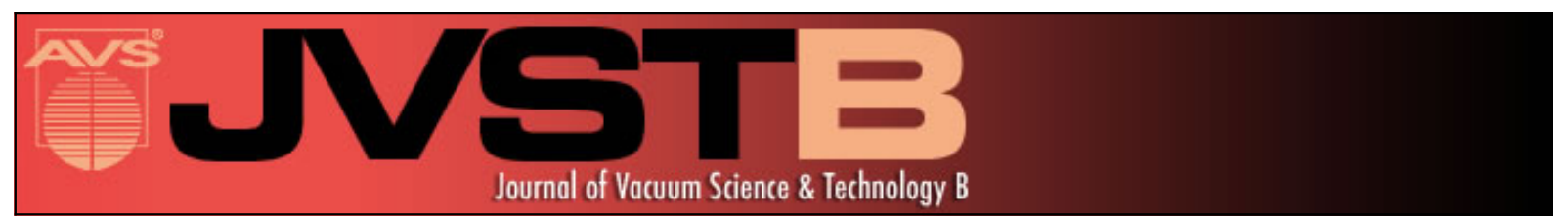

\title{
Electroless deposition of metal nanoparticle clusters: Effect of pattern distance
}

Francesco Gentile, Maria Laura Coluccio, Patrizio Candeloro, Marianna Barberio, Gerardo Perozziello, Marco

Francardi, and Enzo Di Fabrizio

Citation: Journal of Vacuum Science \& Technology B 32, 031804 (2014); doi: 10.1116/1.4870058

View online: http://dx.doi.org/10.1116/1.4870058

View Table of Contents: http://scitation.aip.org/content/avs/journal/jvstb/32/3?ver=pdfcov

Published by the AVS: Science \& Technology of Materials, Interfaces, and Processing

\section{Articles you may be interested in}

Spontaneous decoration of Au nanoparticles on micro-patterned reduced graphene oxide shaped by focused laser beam

J. Appl. Phys. 117, 054304 (2015); 10.1063/1.4906043

Dimensional scale effects on surface enhanced Raman scattering efficiency of self-assembled silver nanoparticle clusters

Appl. Phys. Lett. 105, 073105 (2014); 10.1063/1.4893373

Control of the structure and density of silver nanoparticles obtained by laser-induced chemical deposition from liquids

J. Vac. Sci. Technol. B 31, 06F303 (2013); 10.1116/1.4824328

Controllable aggregates of silver nanoparticle induced by methanol for surface-enhanced Raman scattering Appl. Phys. Lett. 101, 173109 (2012); 10.1063/1.4764024

Noble metal nanoparticle patterning deposition using pulsed-laser deposition in liquid for surface-enhanced Raman scattering

Appl. Phys. Lett. 89, 153124 (2006); 10.1063/1.2359289

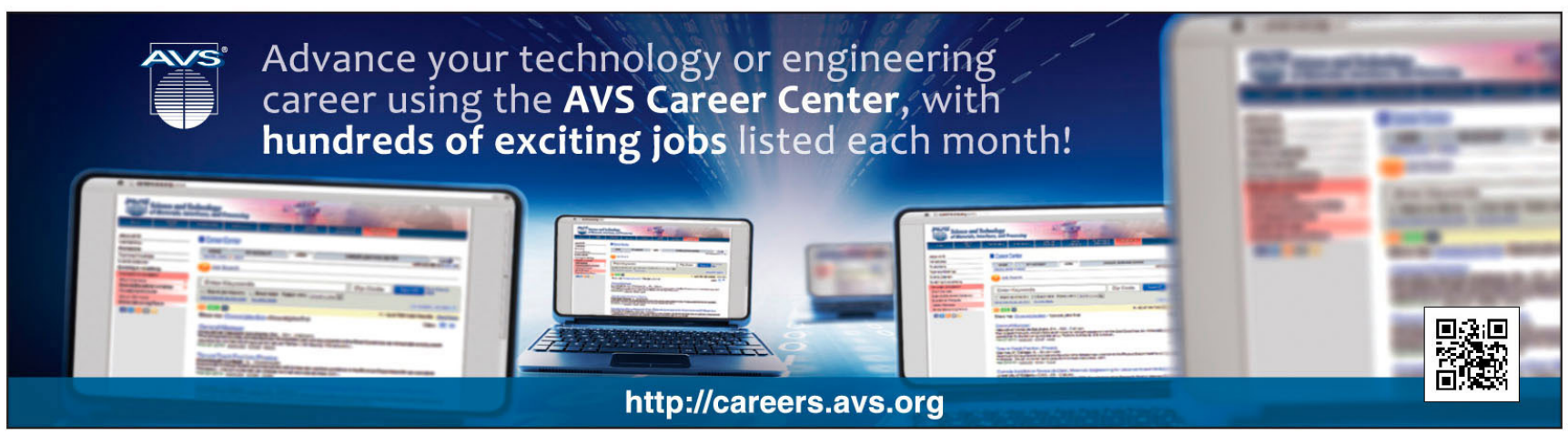




\title{
Electroless deposition of metal nanoparticle clusters: Effect of pattern distance
}

\author{
Francesco Gentile ${ }^{\text {a) }}$ \\ BioNEM (Bio Nano Engineering and Technology for Medicine), University Magna Graecia \\ of Catanzaro-Catanzaro 88100, Italy and Istituto Italiano di Tecnologia, Via Morego 30, \\ 16163 Genova, Italy \\ Maria Laura Coluccio and Patrizio Candeloro \\ BioNEM (Bio Nano Engineering and Technology for Medicine), University Magna Graecia \\ of Catanzaro-Catanzaro 88100, Italy \\ Marianna Barberio \\ Department of Physics, Università della Calabria, Via P. Bucci 33c, 87036 Rende, Italy \\ Gerardo Perozziello \\ BioNEM (Bio Nano Engineering and Technology for Medicine), University Magna Graecia \\ of Catanzaro-Catanzaro 88100, Italy \\ Marco Francardi \\ King Abdullah University of Science and Technology (KAUST), Thuwal 23955-6900, \\ Kingdom of Saudi Arabia

\section{Enzo Di Fabrizio} \\ King Abdullah University of Science and Technology (KAUST), Thuwal 23955-6900, \\ Kingdom of Saudi Arabia and BioNEM (Bio Nano Engineering and Technology for Medicine), \\ University Magna Graecia of Catanzaro-Catanzaro 88100, Italy
}

(Received 14 August 2013; accepted 17 March 2014; published 3 April 2014)

\begin{abstract}
Electroless plating is a deposition technique in which metal ions are reduced as atoms on specific patterned sites of a silicon surface to form metal nanoparticles (NPs) aggregates with the desired characteristics. Those NPs, in turn, can be used as constituents of surface enhanced Raman spectroscopy substrates, which are devices where the electromagnetic field and effects thereof are giantly amplified. Here, the electroless formation of nanostructures was studied as a function of the geometry of the substrate. High resolution, electron beam lithography techniques were used to obtain nonperiodic arrays of circular patterns, in which the spacing of patterns was varied over a significant range. In depositing silver atoms in those circuits, the authors found that the characteristics of the aggregates vary with the pattern distance. When the patterns are in close proximity, the interference of different groups of adjacent aggregates cannot be disregarded and the overall growth is reduced. Differently from this, when the patterns are sufficiently distant, the formation of metal clusters of NPs is independent on the spacing of the patterns. For the particular subset of parameters used here, this critical correlation distance is about three times the pattern diameter. These findings were explained within the framework of a diffusion limited aggregation model, which is a simulation method that can decipher the formation of nanoaggregates at an atomic level. In the discussion, the authors showed how this concept can be used to fabricate ordered arrays of silver nanospheres, where the size of those spheres may be regulated on varying the pattern distance, for applications in biosensing and single molecule detection. (C) 2014 American Vacuum Society. [http://dx.doi.org/10.1116/1.4870058]
\end{abstract}

\section{INTRODUCTION}

The aim of this paper is to investigate the effect of the pattern distance on the overall growth of electroless metal nanoparticle (NP) aggregates. At this purpose, we utilized a combined experimental and theoretical approach, where the theoretical approach is based on a diffusion limited aggregation (DLA) model, while the experimental approach couples electron beam lithography and electroless deposition to obtain clusters of metal nanoparticles with the desired characteristics.

a)Electronic mail: gentile@unicz.it
Nanotechnology is a new science concerned with the study and the fabrication of devices whose size is, in at least a dimension, comprised in the nanometer range. ${ }^{1}$ On account of their small scales, similar devices may directly interact with biological objects (from cells, as large as ten microns, to biomolecules or viruses, sizing few nanometers or less), paving the way to breakthrough strategies in fields like medicine and biotechnology, ${ }^{2}$ for a variety of applications which include, but are not restricted to, the early detection and diagnosis of cancers or other pathologies. ${ }^{3}$

Surface enhanced Raman scattering (SERS) substrates represent an important subset of early detection platforms. ${ }^{4}$ SERS substrates are typically made of nanostructured metal 
materials, at the close proximity of which the electromagnetic (EM) field is locally amplified because of the resonant interaction with the surface plasmons in the metal. ${ }^{5}$ The EM signal may be amplified by several orders of magnitude, and this permits to augment the efficiency of Raman and other spectroscopies, to recognize biological analytes in heretofore unattainable abundance ranges. ${ }^{4}$ The efficiency of SERS depends upon the size and shape (that is, the geometry) of the metal nanostructures, which interact with the EM radiation. ${ }^{6}$ Therefore, understanding and controlling the organization of atoms into ordered systems can possibly improve the performance of metal nanomaterials as efficient SERS devices. The correct understanding of similar mechanisms represents the new frontier in materials science. ${ }^{7-10}$ For all this, there is an ever increasing interest in investigating processes that may manipulate matter at the molecular, nanoparticle, and colloidal level to create nanostructures with the desired characteristics.

In these regards, recently, a promising fabrication technique has been proposed for the formation of aggregates of metal nanoparticles in which the size and shape of these aggregates can be controlled with good accuracy. ${ }^{11}$ The cited technique is based on a multistage procedure where, at a first stage 1, a patterning technique (that is, electron beam lithography) is utilized to prepare the motif where, and at a second stage 2 , metal atoms are deposited using an innovative electroless deposition process. ${ }^{11}$ This breakthrough technique couples bottom-up and top-down approaches to afford exact control over the shape and size of bi- and three-dimensional metal patterns at the nanoscale.

Electroless plating is a deposition technique in which metal ions are reduced as atoms on specific patterned sites of a silicon surface. In Figs. 1(a) and 1(b), we show the preparation of a similar pattern, which is realized using high resolution electron beam lithography. The electron beam exposes the sensitive resist (that is, PMMA) spun on the silicon substrate, to create an array of holes in which the size and distance of those holes may be arbitrarily regulated. Figure 1(c) shows a magnified portion of the cited pattern upon the removal of the resist from the holes. In this configuration, the portion of fresh silicon substrate exposed to growth (that is, the electroactive deposition sites) is limited to the pattern of circles, while the residual PMMA prevents the deposition of metal ions in the remaining parts of the substrate. Therefore, Fig. 1(c) shows an ensemble of diffusing metal ions in the batch solution, where these ions are candidate for being deposited, via electroless, on the circular patterned sites. In Fig. 1(c), we clearly distinguish between the inert PMMA resist, where no deposition may occur, and the active silicon substrate, where instead silver ions may react to form clusters of those ions. In Fig. 1(d), we show a schematic representation of a growth stopped at the early time of the process, which is why the patterns look partially empty. The figure is purely illustrative: in this case, we show a cluster of isolated nanoparticles. Depending on the conditions of growth, one can obtain either compact or discontinuous/fractal structures.

In electroless growth, metal deposition is realized by means of a direct redox reaction that takes place between
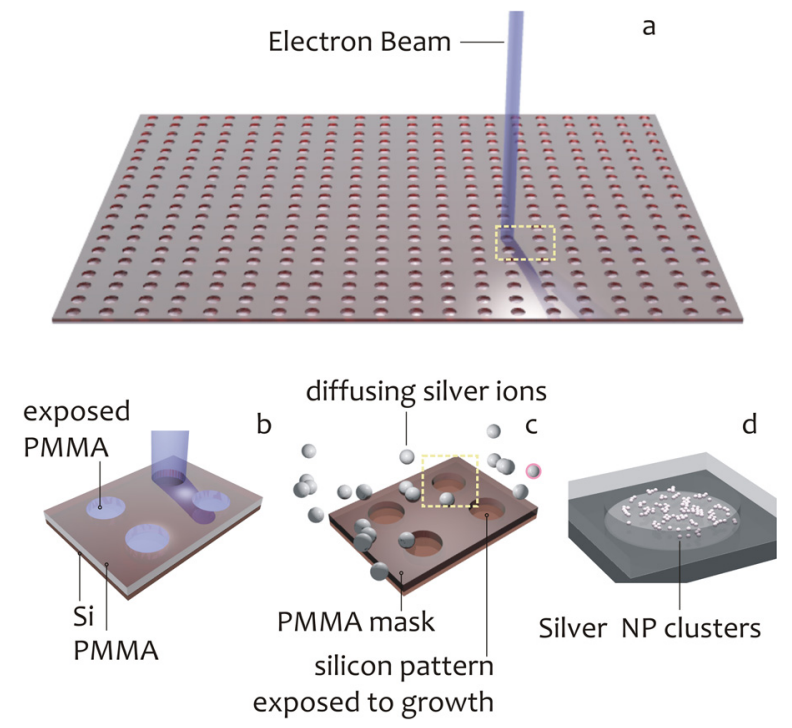

FIG. 1. (Color online) In realizing metal nanostructures, here a technique is used that couples bottom-up and top-down approaches to afford exact control over the shape and size of bi- and three-dimensional patterns. In (a) and (b), we show the preparation of a pattern realized using high resolution electron beam lithography. The electron beam exposes the sensitive resist (that is, PMMA) spun on the silicon substrate, to create a pattern of holes in which the size and distance of those holes may be regulated arbitrarily. (c) A magnified portion of the cited pattern upon the removal of the resist from the holes (and from the holes solely). In this configuration, the portion of fresh silicon substrate exposed to growth (that is, the electroactive deposition sites) is limited to the pattern of circles, while the residual PMMA prevents the deposition of metal ions in the remaining parts of the substrate. Therefore, (c) shows an ensemble of diffusing metal ions in the batch solution, where these ions are candidates for being deposited, via electroless, on the circular patterned sites; we may clearly distinguish between the inert PMMA resist, where no deposition may occur, and the active silicon substrate, where instead silver ions may react to form clusters of those ions. In (d), we show a schematic representation of a growth stopped at the early time of the process, which is why the patterns look partially empty. The figure is purely illustrative; in this case, we show a cluster of isolated nanoparticles. Depending on the conditions of growth, one can obtain either compact or discontinuous/fractal structures.

the silver ions and the silicon to form the desired nanoparticles. $^{12,13}$

In Ref. 11, this scheme was utilized for realizing silver nanolenses, where an exquisite control of the geometry is the key attribute of a device that is capable of amplifying the electromagnetic radiation for applications in SERS spectroscopy.

In the formation of the super-clusters of metal atoms, the kinetics of reaction is nearly two orders of magnitude faster than diffusion, which can be therefore disregarded. This implies that a generic electroless aggregate can be correctly described by a stochastic, DLA model ${ }^{12,14,15}$ where ions, undergoing a random walk due to Brownian motion, cluster together to form aggregates of such ions, revealing a fractal motif. ${ }^{14,16}$ DLA founds upon the paradigm that, in the limit of very fast chemical reactions, diffusion is the sole driving force that regulates the dynamics of aggregation of NPs.

DLA is a simulation method based on simple algorithms, which is capable of providing valuable help in understanding natural process, in alternative to models that instead make use of analytical solution of equations. ${ }^{17}$ Indeed, in many 
situations, it is possible to model a system by entities that diffuse and occasionally react upon encounter each other. These systems are governed by two time scales: the diffusion time, which is the characteristic time required for two particles to meet, and the reaction time, which is the characteristic time for particles to react when held in proximity (within the reaction range) to each other. When the diffusion time dominates over the reaction time, the process is limited by diffusion. DLA is formally regulated by certain rules, which can be found, for example, in Refs. 14 and 16, and are here recapitulated in Sec. II. Proposed for the first time in the pioneering works of Witten and Sander in the early 1980s, ${ }^{16}$ this numerical framework is very well understood and has been utilized, to name a few examples, to reproduce the process of electrodeposition in single wells, ${ }^{7}$ to investigate the role of sedimentation and buoyancy on the kinetics of colloidal aggregation, ${ }^{18}$ to describe solute percolation in soils, ${ }^{19}$ to describe the early stages of thin-film growth at an atomistic scale,${ }^{20}$ and to gain insight into physical phenomena including the annealing of defects in crystals ${ }^{21}$ and the electron hole recombination. ${ }^{22}$

In a recent work, ${ }^{14}$ we utilized a similar DLA approach to describe the nature of electroless metal nanomaterials in aggregates in which the size of the patterns was varied over a significant range. The present manuscript broadens this DLA model to include the effect of pattern distance in the analysis; therefore, it expands and improves the scheme introduced in Ref. 14. On changing the distance $\delta$ between the patterns of silicon exposed to metal ions deposition (Fig. 2 ), we observed that the packing factor $\kappa$ of the aggregates increases with $\delta$ in the low distance range (that is, smaller than three times the diameter); instead, when the distance is sufficiently large (that is, larger than three times the diameter), $\kappa$ settles down to a constant value. Therefore, an interparticle spacing corresponding to three times the pattern size is a limiting value of distance below which the inference of adjacent groups of metal nanoparticle clusters would impair the efficiency of growth, and above which the growth would be unaffected by changing $\delta$. These findings were supported by direct experiments. Utilizing convenient experimental conditions, we obtained a range of aggregates of silver atoms and particles with varying pattern distance; lately, we imaged those aggregates using atomic force and scanning electron microscopy. We introduced nonconventional variables, including the fractal dimension and the packing factor, to realize a comparison between theory and experiments on a quantitative basis. In doing this, we found a good match between experiments and theory.

In the discussion, we showed how this concept can be used to fabricate ordered arrays of silver nanospheres, where the size of those spheres may be regulated on varying the pattern distance, for applications in biosensing and single molecule detection.

\section{MODELING}

\section{A. Using DLA simulations to reproduce particle growth}

The mechanism of metal growth was reproduced under the assumption of a DLA process. This assumption is true when diffusion dominates over chemical reaction or, equivalently, when the kinetics of metal reduction is extremely
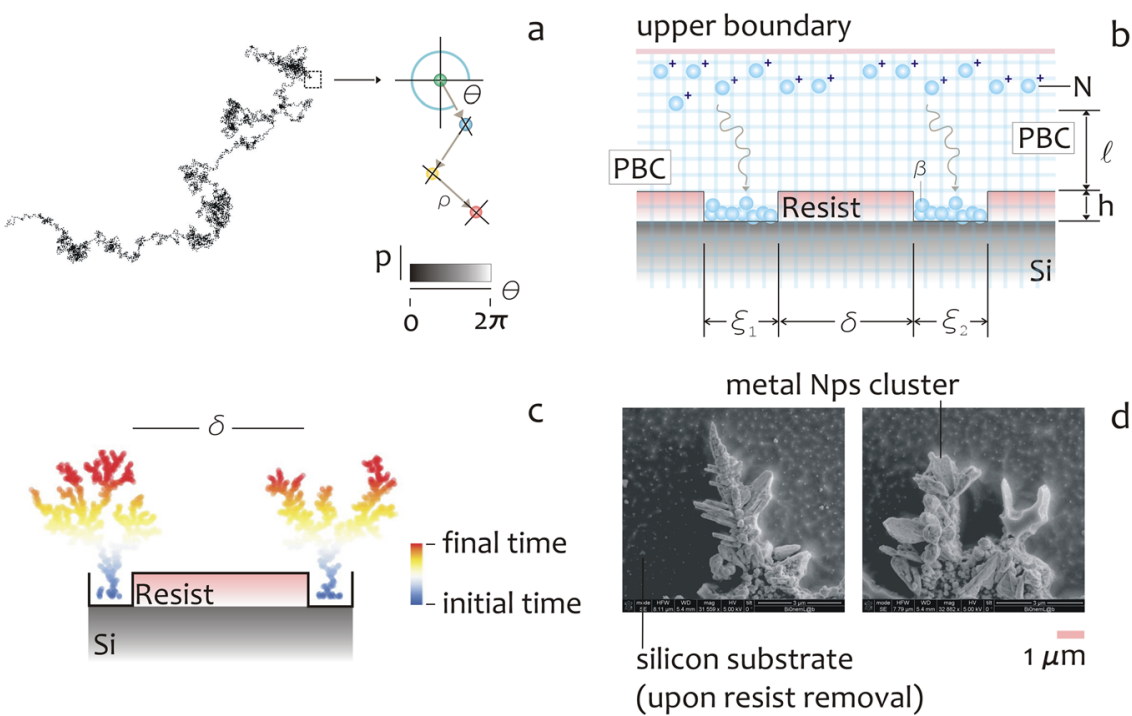

FIG. 2. (Color online) (a) Particle moving a stochastic, random walk. At any step, the probability distribution of the direction of movement is uniform or flat, with events equally likely to occur anywhere within the interval from 0 to $2 \pi$. (b) Schematic representation of the DLA process and typical pattern of migration of a particle. (c) An example of a typical numerical DLA growth in a pattern constituted by a couple of electroactive deposition sites, where the dimensions of the atoms has been exaggerated for illustrative purposes. In practice, (c) illustrates the final result of a simulated electroless deposition process, realized in the same system reproduced in (b). On the fresh silicon substrate, ions may cluster to form isolated structures, or complex geometries. The complex, random (Brownian) nature of the DLA model here utilized, is responsible for the fractal nature of these numerical aggregates. Under certain experimental conditions, real electroless deposits reveal a clear multibranched structure, which recalls the DLA aggregates. This is the case of the SEM images in (d), where the temperature and time of deposition were arbitrarily chosen to overboost the electroless reaction: for this configuration, the process yields a hierarchal structure, where multiple particles are present in a single active site. 
fast. The model makes it possible to decipher, at an atomic level, the rules governing the evolution of the growth front, and to explore ways to tailor film morphology to obtain specific characteristics. ${ }^{16,20}$

The choice of using here a DLA model, where diffusion dominates over chemical reactions, is motivated by a number of observations, widely reported in literature, where electroless method produces dendritic nanostructures, with stems, branches, and leaves. ${ }^{15,23}$ These fractal structures are generally observed in nonequilibrium growth processes, and DLA is one of the most suitable approach for their description. $^{12,15,24}$

The model utilized here speculates that, if a diffusing atom sticks to the aggregate where it hits the aggregate, then fractal geometries will be formed. It neglects mechanisms such as 1) adatom diffusion on terraces and nucleation of islands or 2) diffusion along island edges, a detailed description of which is instead included in Ref. 20. Therefore, the introduced model is a simplified hit and stick DLA (which is sometimes called, after Zhang et al., ${ }^{20}$ regime I of fractal like growth, with zero local relaxation), where it is assumed that surface diffusion is much slower that the diffusion of silver ions in the bulk solution (or, alternatively, that surface diffusion is hindered by the large barrier between the sites). DLA may be improved to include the effect of diffusion on the surface, simply decreasing the probability $p$ of sticking upon contact, that in the present configuration is fixed as $p=1$ (that is, a deterministic process). In doing so, we would obtain an extended fractal growth (or regime II of DLA) where (differently from the regime I) the thickness $b$ of the branches in a fractal set would be well larger than one atom (and thus, $b>1$ ). In our model, $p$ is an explicit variable, which was voluntarily set as $p=1$ for computational convenience. Nevertheless, it may be arbitrarily changed to assume any real value comprised between 0 and 1 to relax the constraint of a zero local relaxation (in this new regime II, an atom arriving at the edge of an island would have additional time to relax to a more favorable site before it is pinned in the position by the arrival of another atom). This more sophisticated version of DLA model would lead to more compact aggregates of atoms compared to the fractal, strongly anisotropic numerical clusters that are shown in the present paper.

Despite this, the basic DLA model we used here revealed itself effective in capturing the basic mechanism of particle growth as a function of pattern distance, as largely demonstrated in the results presented in the paper. Any further evaluation of the model can possibly lead to a more accurate description of the evolution of the growth front over time. Nevertheless, this understanding goes beyond the purpose of the present paper and is left for future work.

In our model, the displacement of a metal ion, at any time, is arbitrary, and thus, the trajectory of the ions can be correctly described by a random walk, as to resemble a Brownian motion [Fig. 2(a)]. Brownian motion is a continuous-time probabilistic process: its use here is motivated by mathematical convenience and the accuracy of the model to describe an otherwise complex phenomenon.
At very short time scales, the motion of a ion undergoing Brownian motion is dominated by its inertia and its displacement will be linearly dependent on time, $\Delta x=v \tau$. This can be reproduced or simulated in a regular grid [Fig. 2(b)] where ions are dislodged by the finite distance $\Delta x$ in the time interval $\tau$, which is the mean time between collisions. The instantaneous velocity of the ions, $v$, is maintained constant during $\tau$, and it would depend solely upon the energy of the system. The distribution of displacements probability of the Brownian particle itself is best described using a Gaussian density function, centered around the origin of the walk, which means that the most probable position of a ion after a sufficiently large number of steps is paradoxically its original position. The root mean square distance of the walk gives a measure of the extent of spread of the ion ensemble, and this would be proportional to time ${ }^{25}$

$$
\left\langle r^{2}\right\rangle=\left\langle x^{2}\right\rangle+\left\langle y^{2}\right\rangle=4 D t,
$$

where $x, y$, and $r$ are the Cartesian coordinates in the plane, while the celebrated Stokes-Einstein equation may be used to derive the diffusion coefficient $D$ (Ref. 26)

$$
D=\frac{k_{b} T}{6 \pi \mu a},
$$

where $k_{b}$ is the Boltzmann constant, $T$ the absolute temperature of the system, $\mu$ the viscosity of the medium, and $a$ is the diameter of an ion with mass $m$. $D$ can be alternatively expressed in terms of $\Delta x$ and $\tau$ as

$$
D=\frac{\Delta x^{2}}{2 \tau}=\frac{(v \tau)^{2}}{2 \tau}=\frac{k_{b} T}{2 m}
$$

where the kinetic theory of gases has been used for obtaining the right hand term of Eq. (3). ${ }^{25}$ Notice that, on comparing Eqs. (2) and (3), given the results of the simulations, the behavior of the real system would be determined. For the present configuration, the variables of the system, including the temperature $T$, the mass $\mathrm{m}$ and diameter $a$ of the dislodging ions, and the viscosity of the medium, assume prescribed values as recapitulated in Table I.

This concept was used here to simulate the deposition of metal ions in a patterned silicon substrate, where the number of patterns is restricted to two, and their distance can vary

TABLE I. Physical variables and values thereof of the silver ion gas system considered for the particle growth.

\begin{tabular}{lcccc}
\hline \hline Variable & Symbol & Units & Value & Source \\
\hline Mass of a silver ion & $\mathrm{m}$ & $\mathrm{kg}$ & $1.7810^{-25}$ & Ref. 48 \\
Van der Waals size of a silver ion & $\mathrm{R}$ & $\mathrm{m}$ & $117210^{-12} \mathrm{~m}$ & Ref. 48 \\
Temperature of the system & $\mathrm{T}$ & $\mathrm{k}$ & 323 & - \\
Velocity of the ion & $\mathrm{v}$ & $\mathrm{m} \mathrm{s}^{-1}$ & 22 & $\sqrt{k_{b} T / m}$ \\
Viscosity of water at T $=323 \mathrm{~K}$ & $\mu$ & $\mathrm{Pa} \mathrm{s}$ & $0.310^{-3}$ & Ref. x \\
Diffusion coefficient & $\mathrm{D}$ & $\mathrm{m}^{2} \mathrm{~s}$ & $1.510^{-9}$ & $k_{b} T / 6 \pi \mu a$ \\
Mean path length & $\Delta \mathrm{x}$ & $\mathrm{nm}$ & 0.5 & $4 D / v$ \\
Time interval & $\tau$ & $\mathrm{s}$ & $2.210^{-11}$ & $\Delta x / v$ \\
\hline \hline
\end{tabular}


over a significant range. Consider, for ease of visualization, the scheme in Fig. 2(b). At a distance $l$ from the wells, $N$ ions are simultaneously released in the system, where $N$ should be chosen with care to reproduce the initial concentration of silver ions. At any cycle, the particles move within a regular square pattern of cells by one lattice unit (l.u.), and thus, the mean path length is $\Delta x=1$ (l.u.). At the side boundaries (which are, the left and the right) of the domain, periodic boundary conditions are imposed, and when an individual ion passes through one of those faces, it instantaneously reappears on the opposite face with the same velocity. In topological terms, the plane can be thought of as being mapped onto a torus; by doing so, an infinite perfect tiling of the system is simulated in the longitudinal direction, and this accounts for the fact that the area of the wells is small compared to the area above the wells. At the upper boundary of the system, and at the side walls of the wells, a bouncing condition is imposed, whereby the ions which collide with those boundaries would rebound in an opposite direction. At the lower border of the system: the seeds $\xi_{1}, \xi_{2}$ represent the regions of fresh silicon substrate exposed to growth. Their distance $\delta$ can be conveniently adjusted according to the problem at study. Therefore, $\xi_{1}+\xi_{2}$ is a discontinuous line of nucleation sites. The structure of the aggregate $\beta(t)$ would depend upon $\delta$ as explained in Sec. IV. When an ion migrates to touch $\xi_{1}$ or $\xi_{2}$, it stops, it is incorporated by either $\xi_{1}$ or $\xi_{2}$, and then the size of the overall aggregate is augmented by one unit. Simultaneously, a new particle is instantaneously created and randomly positioned in the upper region of the domain; in doing so, the total number of ions $n$ is kept constant during the process. After a certain number of repetitions, a binary system of aggregates is formed as in Fig. 2(c). The multibranched arrangement of particles recalls the dendrite, fractal nature that electroless grown systems reveal under certain growth conditions [Fig. 2(d)].

Therefore, in Fig. 2(c), we show an example of a typical numerical DLA growth in a pattern constituted by a couple of electroactive deposition sites, where the dimensions of the atoms has been exaggerated for illustrative purposes. DLA may correctly reproduce the real electroless process solely if the metal ions are deposited on specific sites of the substrate, being the silicon regions from which the PMMA resist has been removed.

On the fresh silicon substrate, ions may cluster to form isolated structures or complex geometries. The complex, random (Brownian) nature of the DLA model is responsible for the fractal nature of these numerical aggregates. Under certain experimental conditions, real electroless deposits reveal a clear multibranched structure that recalls the DLA aggregates. This is the case of the SEM images in Fig. 2(d), where the temperature and time of deposition were arbitrarily chosen to over-boost the electroless reaction: for this configuration, the process yields a hierarchal structure, where multiple particles are present in a single active site.

For the configurations considered in the work, the parameters of the simulations were adjusted as follows. The simulation was halted after a number of iterations $N i=O\left(10^{10}\right)$. The total number of ions in the solution $N$ and the length of each seed were hold fixed, being $N=2000$, length $\left(\xi_{1}\right)$ $=$ length $\left(\xi_{2}\right)=25$ (l.u.). The distance between the seeds $\delta$ was steadily changed from 25 to 100 (1.u.) (that is, from 1 to 4 times the length of each seed). Considering that the mean path length is about $\Delta x \sim 1 / 2 \mathrm{~nm}$ (Table I), the scale factor of the problem is 2 , and thus 25 (l.u.) would roughly correspond to $12.5 \mathrm{~nm}$ in the real system.

The structure of clusters of occupied lattice sites [Fig. 2(c)] exhibits geometric scaling relationships, which are characteristic of fractals and can be used to estimate an effective fractal dimensionality $D f$, which has a value of about $5 / 3$. The fractal dimension is a parameter that can be used to describe intimately the topography of a variety of systems, especially at the nanoscales. The concept of fractal dimension, and the techniques needed to derive it, are recapitulated in Ref. 27 and are recalled here in the following of the Modeling section. The importance of $D f$ resides in the fact that it is used in the definition of certain parameters or properties which describe the deposit.

As for an instance, the thickness $Q$ of the aggregate scales with the total number of deposited atoms $n$, as ${ }^{28}$

$$
Q \sim n^{1 /\left(D_{f}-1\right)},
$$

the fractal dimension is also correlated to the distribution or number $I$ of clusters with size $S$ as a function of cluster $\operatorname{size}^{28}$

$$
I(S) \sim S^{-\left(1+\frac{1}{D_{f}}\right)} .
$$

Most importantly, the mean cluster size can be deduced and correlated with $n$ via a very simple power law, being ${ }^{29}$

$$
S \sim n^{D_{f} /\left(D_{f}-1\right)},
$$

and thus, given $n$ that can be easily calculated, the mean cluster size would be readily derived. The packing factor $\kappa$ can be therefore obtained as the ratio of $S$ to the size of a single pattern, $\kappa=S / d$. In the following, $\kappa$ is determined and compared for numerical and experimental particles assemblies as a function of the inter-pattern distance $\delta$.

\section{B. Deriving the fractal dimension of the DLA aggregates}

Fractals are mathematical objects that are too irregular to be described by conventional geometry. They all retain, to different extents, certain properties that may be reviewed as follows: 1) they reveal details on arbitrarily small scales (fine structure); 2) they can be generated (and thus described) by short algorithms (perhaps recursively); 3) they exhibit a fractal dimension $D f$ strictly greater than the classical topological dimension. ${ }^{30}$ The fractal dimension of the aggregates was derived from their characteristic power spectrum density function $C(q)$.

The power spectrum delivers significant information regarding the microstructure of the systems at study. In the case of self-affine structures, for which a rescale in the 
planar coordinates $x \rightarrow b x$ and $\mathrm{y} \rightarrow$ by is accompanied by a rescaling in the normal direction $z(b f) \rightarrow b H z(f)$, the power spectrum $C(q)$ takes the form ${ }^{27}$

$$
C(q)=\frac{H}{2 \pi}\left(\frac{h_{o}}{q_{o}}\right)^{2}\left(\frac{q}{q_{o}}\right)^{-\beta},
$$

where $q$ is the wave number, related to the characteristic wavelength $\lambda$ as $q=2 \pi / \lambda$; $q_{o}$ is the lower cut-off wavenumber corresponding to an upper cut-off wavelength $\lambda_{\mathrm{o}}=2 \pi / q_{o}$; $h_{o}$ is related to the rms roughness amplitude as $h_{o}=2^{1 / 2} \mathrm{rms}$; $H$ is known as the Hurst coefficient. In a log-log plot, the power spectrum density appears as a line with a slope $\beta$ for $\mathrm{q}$ $>\mathrm{q}_{\mathrm{o}}$. The slope $\beta$ is related to the Hurst parameters as $\beta=2(H+1)$ for bidimensional structures, and $\beta=1+2 H$ for monodimensional structures. The fractal dimension $D f$ of the surface can be derived from $H$ as $D f=3-H$ and $D f=2-H$, for bi- and monodimensional structures, respectively. Therefore, if one can determine the power spectrum of a set of points, the corresponding fractal dimension would be readily derived from a simple graphical procedure.

The power spectrum of DLA aggregates, in turn, can be derived from a pair correlation analysis. The pair correlation function $g(r)$ is related to the probability of finding the center of a particle at a given distance $r$ from the center of another particle. For short distances, this is related to how the particles are packed together. Here we provide a simple algorithm following which $g(r)$ was calculated.

One should choose and fix a value of $d r$ sufficiently small. Therefore, for all the values of $r$ considered for the analysis, the following steps of a procedure should be repeated: 1) count the particles positioned at a distance comprised between $r$ and $r+d r$ from the reference particle. Those are all particles in a circular shell, with thickness $d r$, surrounding the reference particle. 2) Divide your total count by the number of reference particles you considered. 3) Divide this number by $2 \pi r d r$, that is, the area of the circular shell. This accounts for the fact that as $r$ gets larger, for trivial reasons you find more particles with the given separation. 4) Divide this by the particle number density. This ensures that $g(r)=1$ for data with no structure. 5) The resulting value is the value of the pair correlation function at the specific distance $r, g(r)$.

\section{EXPERIMENT}

Silver NPs (and thus clusters of silver atoms) and aggregates of silver NPs (and thus super-clusters of silver atoms) were obtained, in which the spacing of the patterns was varied over a significant range. The diameter $d$ of the patterns was instead hold fixed, being $d=10 \mu \mathrm{m}$, which is sufficiently large to have a statistically significant number of nanometric grains during the growth. An electroless deposition method was utilized to form nanoparticulates with the desired characteristics. The internal structure of those aggregates was analyzed through direct SEM and AFM imaging; from those images, in turn, certain variables were extracted, including the average cluster size and the ratio of the average cluster size to the pattern diameter, to yield objective information the topography of the systems at study. Therefore, this information was explained within the framework of a DLA model. Hydrofluoric (HF) acid and silver nitrate $\left(\mathrm{AgNO}_{3}\right)$ were purchased from Carlo Erba. Deionized water was used for all experiments. All chemicals, unless mentioned otherwise, were of analytical grade and were used as received.

\section{A. Fabrication of silver NPs aggregates}

Clusters of metal NPs were realized. These are cylindrical patterns or holes of fresh silicon, with a variable pitch and a constant diameter, which were exposed to electroless deposition for the formation of silver NPs aggregates. (100) silicon wafers (Jocam, Milan, Italy) were cleaned with acetone and isopropanol to remove possible contaminant and then etched with a $4 \%$ wet HF solution. The wafers were then rinsed with D.I. water and dried with $\mathrm{N}_{2}$. Standard optical lithography techniques (Karl Suss Mask Aligner MA 45, Suss MicroTec GA, Garching, Germany) were utilized to obtain variable spacing disks within a layer of positive resist (S1813, Rohm and Haas) that was spin-coated onto clean silicon wafers. The sample was then dipped in a $0.15 M \mathrm{HF}$ solution containing $1 \mathrm{~m} M$ silver nitrate $\left(\mathrm{AgNO}_{3}\right)$ for $60 \mathrm{~s}$ at a constant temperature $T=313 \mathrm{~K}$. By doing so, Ag-metal particles were deposited on the lithographically predefined areas (which are the cylindrical cavities as described above) where the reduction of oxidized metallic species produced the formation of neutral metal atoms. ${ }^{11}$ The device was then washed with water at room temperature to stop the reaction and with D.I. water at $50^{\circ} \mathrm{C}$. Finally, the sample was dried under nitrogen flux. The masks necessary for optical lithography were fabricated using standard electron beam lithography (Crestec CABL-9000C Electron beam lithography system) methods, using batch files conveniently generated on the basis of a criterion of rational design described in Ref. 31.

In growing the intended structures, the total silicon surface exposed to growth is maintained constant in different experiments. For every considered configuration, this area, and with that the total volume of the wells, is small compared to the volume of solution. Therefore, electroless deposition always takes place in the presence of an excess of solute (that is, metal ions), regardless the total area of the pattern interested to growth. What are really important are the local concentrations effects, that is, to which extent metal ions are available to growth in close proximity of a pattern. Consider Figure 7 in Appendix A for ease of visualization. Every pattern (well) would have a certain number $N$ of metal ions immediately above and beside it, comprised in certain region of interest. These particles represent the candidates for being deposited in a single pattern in a given amount of time. When the patterns are sufficiently close, the cited regions of interest would to a certain extent superimpose, and thus, the free particles available for growth would reduce per each pattern. This would depend on the pattern distance in the paper; we unreveal in Fig. 5(e) this dependence. 


\section{B. Sample SEM characterization}

SEM images of the samples were captured using a Dual Beam (SEM-FIB) - FEI Nova 600 NanoLab system. During the acquisitions, beam energy of $15 \mathrm{keV}$ and corresponding electron current of $0.14 \mathrm{nA}$ were used. The clusters of NPs were imaged employing the mode 2 configuration, whereby images can be magnified over 2500000 of times and ultrahigh resolution may be achieved.

\section{Sample AFM characterization}

Atomic force microscopy (Veeco MultiMode with NanoScope V controller) was used for the measurements of the nanostructures. All the measurements were performed in a dry environment in intermittent contact mode over a sampling area of $500 \times 500 \mathrm{~nm}^{2}$. Room temperature was hold fixed for all the acquisitions. Ultrasharp $\mathrm{Si}$ probes (ACLA-SS, AppNano) with a nominal tip radius less than $5 \mathrm{~nm}$ were used for high resolution. Multiple measurements were done in different scan directions to prove the avoidance of artifacts. The images had a resolution of $1024 \times 1024$ points and were acquired at a scanning rate of $1 \mathrm{~Hz}$. The images obtained were processed with the WSxM ${ }^{\circledR}$ software, using either flattening or plane fit according to the relief characteristics, with the minimal polynomial order needed. Using conventional mathematical procedures implemented in Mathematica $₫$, the mean cluster size $S$ and packing factor $\kappa$ for each system were determined.

\section{RESULTS AND DISCUSSION}

\section{A. Fabrication of clusters of silver nanoparticles}

The idea that the formation of silver supramolecular structures is influenced by the spacing of those structures originated from the observation that, in growing metal clusters where the pattern distance is varied over a significant range, the morphology of the surface varies with the pattern distance. In Fig. 3, SEM images show silver nanoparticle aggregates in patterns where their distance varies from fractions [that is, $\delta=0.5 d$, Fig. 3(a)] to several times the diameter [that is, $\delta=2 d$, Fig. 3(b), $\delta=2 d$, Fig. 3(c)] of those patterns. The metal grains are disposed in the plane as to form random cluster of nanoparticles, where the size and number of particles in a network varies moving from Figs. 3(a) to 3(c). This is even more evident in Fig. 3(d) (adjacent patterns, $\delta=2 d$ ) and Fig. 3(e) (separated patterns, $\delta=4 d$ ): larger magnification SEM images reveal that the morphology of metal clusters is significantly influenced by the spacing of the patterns in which silver nanoparticles are deposited. To support this intuition, the electroless formation of superclusters of silver nanoparticles was investigated systematically.

Circular patterns were fabricated with a fixed diameter $d=10 \mu \mathrm{m}$, and a pattern distance ranging from one to four times $d$, and thus $1 \leq \zeta \leq 4$, where $\zeta=\delta / d$ is the ratio of the distance to the pattern diameter. In doing so, the diameter of the lithographed pattern is sufficiently large to comprehend a large number of nanograins upon growth. The resulting

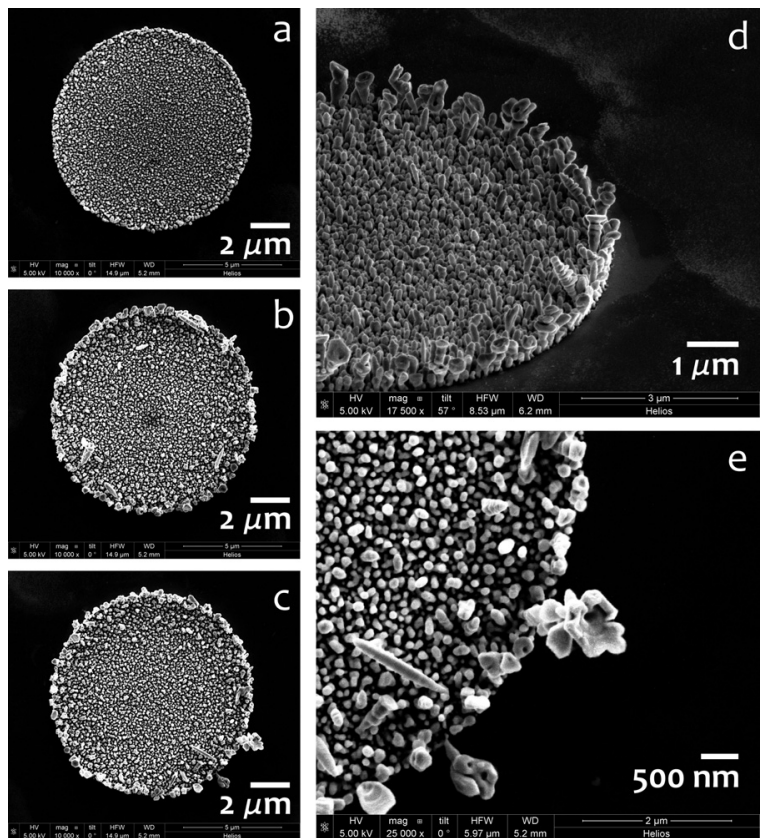

FIG. 3. Idea that the formation of silver supramolecular structures is influenced by the spacing of those structures, originated from the observation that, in growing metal clusters where the pattern distance is varied over a significant range, the morphology of the surface varies with the pattern distance. SEM images show silver nanoparticles aggregates in patterns where their distance varies from fractions $[\delta=0.5 d$, (a) $]$ to several times the diameter $[\delta=2 d$, (b), $\delta=4 d$, (c)] of those patterns. Larger magnification SEM images reveal that the morphology of metal clusters is significantly influenced by the spacing of the patterns where silver nanoparticles are deposited for adjacent patterns $[\delta=2 d,(\mathrm{~d})]$ and separated patterns $[\delta=4 d$, (e)].

structures were therefore verified using direct SEM and AFM imaging and, from the analysis of those images, the average cluster size was derived as a function of the pattern distance.

SEM micrographs reveal details of the nanoparticles aggregates at the smaller scales for either small $[\zeta=1$, Figs. $4(\mathrm{a})$ and $4(\mathrm{~b})]$ and large $[\zeta=4$, Figs. $4(\mathrm{e})$ and $4(\mathrm{f})] \zeta$. For $\zeta>4$, the morphology of the aggregates is fairly independent on $\zeta$, therefore $\zeta=1$ and $\zeta=4$ can be considered as the lower and upper limits of particle growth. On comparing the images in Figs. 4(a) and 4(e) and especially Figs. 4(b) and $4(\mathrm{f})$, where the clusters are imaged with a low $(15000 \times)$ and high $(50000 \times)$ magnification factor, it can be observed that the nanograins are randomly distributed over the lithographed silicon surface, with a cluster size $S$ that increases with the non-dimensional distance $\zeta$ and that, on average, is smaller for $\zeta=1$ compared to $\zeta=4$. Standard image analysis algorithms were utilized to gain quantitative information from those images. The grain size distribution was deduced and reported, in form of a bar chart, for $\zeta=1$ and $\zeta=4$ in Figs. 4(c) and 4(g) respectively. From those distributions, the average cluster size was derived, being $S=82 \pm 4 \mathrm{~nm}$ for the dense close-packing of patterns $(\zeta=1)$, and $S=148 \pm 9 \mathrm{~nm}$ for the scattered-packing of patterns $(\zeta=4)$. Notice that the frequency distributions are skewed (that is, nonsymmetric), whereby the tails on the right side are considerably longer relative to the left side and this means that, for a given configuration, larger particles are less frequent 

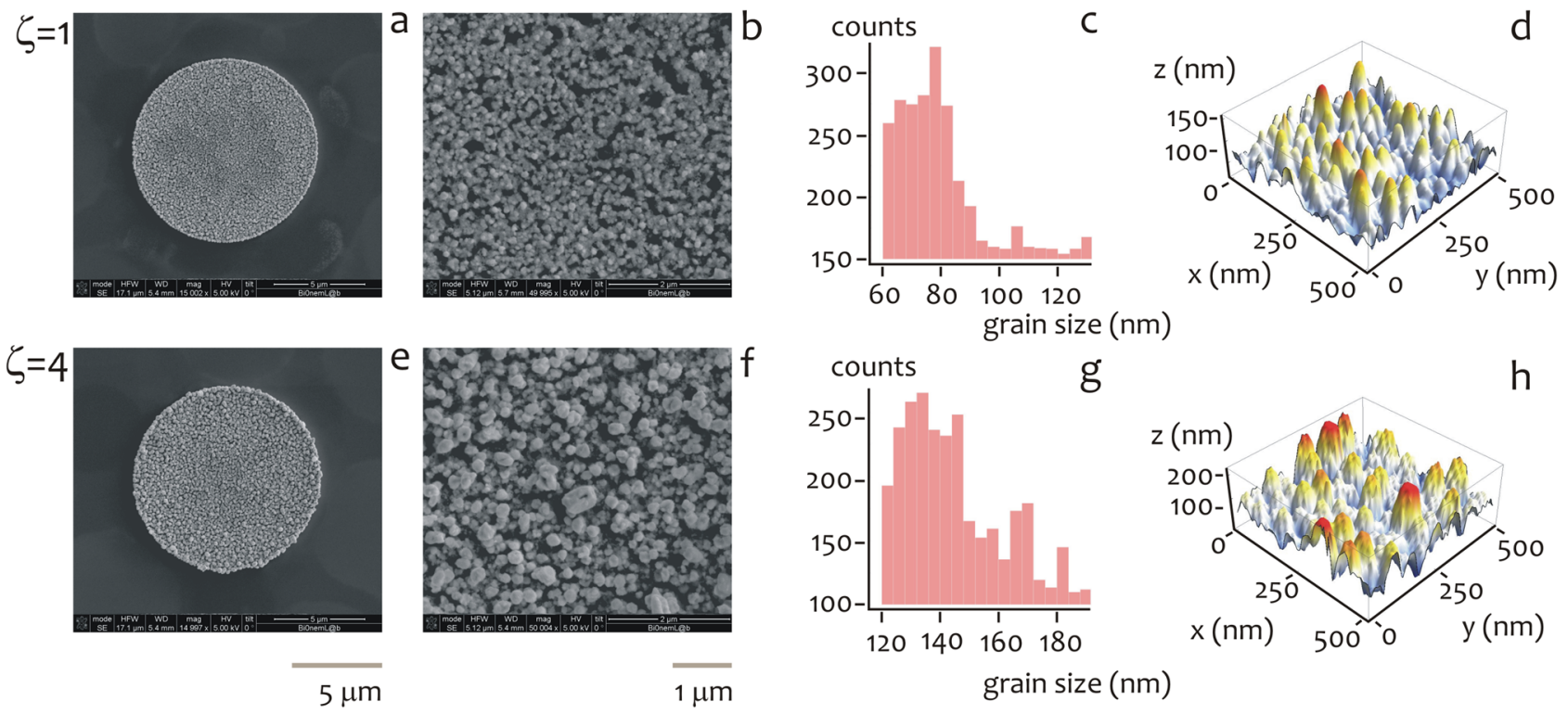

FIG. 4. (Color online) (a) SEM image of nanoclusters of silver NPs obtained over circular patterns in which the spacing of the structures is one time the diameter $\mathrm{d}, \zeta=1$, for this configuration, the diameter of the pattern is $10 \mu \mathrm{m}$ (SEM magnification $15000 \times$ ). (b) SEM image of nanoclusters of silver NPs obtained over circular patterns in which the spacing of the structures is one time the diameter $d, \zeta=1$ (SEM magnification $50000 \times$ ). (c) The grain size distribution reported, in form of a bar chart, for $\zeta=1$. From this distribution, the average cluster size is derived as $S=82 \pm 4$. (d) AFM profile of the silver nanograin clusters $(\zeta=1)$. (e) SEM image of nanoclusters of silver NPs obtained over circular patterns in which the spacing of the structures is four times the diameter $d$, $\zeta=4$ (SEM magnification $15000 \times$ ). (f) SEM image of nanoclusters of silver NPs obtained over circular patterns in which the spacing of the structures is four times the diameter $d, \zeta=4$ (SEM magnification $50000 \times$ ). (g) The grain size distribution reported, in form of a bar chart, for $\zeta=4$. From this distribution, the average cluster size is derived as $S=148 \pm 9$. (h) AFM profile of the silver nanograin clusters $(\zeta=4)$.

than smaller ones. Considering that skewed data often occur due to lower or upper bounds on the data, this effect can be ascribed to the fact that the dimension of the particles must be non-negative, and accordingly, they would have a left/low bound of $0 \mathrm{~nm}$.

The histograms, shown in Fig. 4 for the limiting cases $\zeta=(1,4)$ solely, were derived for all the values of $\zeta$ in the considered range of pattern distances and indicate that the cluster size smoothly transitions from small to large values for increasing pattern distance. Moreover, this effect saturates with $\zeta$, as reported in the table of Fig. 5(d).

Also notice, on observing the clusters of silver nanoparticles deposited on different circular patterned surfaces [Figs. 4(a) and 4(e)], the growth is more pronounced at the borders or edges of those patterns. This effect can be ascribed to a nonuniform distribution of surface energy density, which, at the edges, is larger than on flat surfaces, as explained in Appendix B.

Finally, the AFM profiles of the same aggregates, reported in Fig. 4(d) for $\zeta=1$, and Fig. 4(h) for $\zeta=4$, support the finding that, on growing silver NPs in patterns with variable distance, the efficiency of growth increases with $\zeta$, where with efficiency we mean the tendency of the process to form few, larger, and continuous clusters $(\zeta=4)$, in opposition to more and smaller ones $(\zeta=1)$. This is confirmed by the characteristic packing factor $\kappa$ derived for all the structures under examination and reported in the table of Fig. 5(d). The packing factor is defined as the ratio of the cluster size to the pattern diameter; it gives a measure of the packing density of the systems: the larger $\kappa$, the lower the number of gaps or discontinuities in a pattern.

\section{B. Simulating the formation of clusters of silver nanoparticles}

These results were recapitulated within the framework of a DLA model. Pair aggregates were produced with the pattern distance varying from low (that is, $\zeta=1$ ) to large (that is, $\zeta=4$ ) values of $\zeta$, as explained in the Modeling section. Examples of such aggregates are reported in Fig. 5(a) $(\zeta=1)$ and Fig. 5(b) $(\zeta=4)$. These diagrams demonstrate the dependency of the velocity of particle deposition on $\zeta$, whereby the size of the aggregates $n$ augments for increasing pattern distance. Also notice that the number $I$ of branches or clusters increases for $\zeta$ moving from 1 to 4 . Per each configuration, a pair correlation analysis [Fig. 5(c)] was used to calculate the fractal dimension of those aggregates, that is, $D f=5 / 3$. This dimension, in turn, was used to determine the mean numerical cluster size $S$ [Eq. (6)] and thus the packing factor $\kappa$ that is reported, for all the considered $\zeta$, in the table of Fig. 5(d). The cluster size, for a numerical lattice of particles, has the meaning of the minimum correlation distance defining subsets of those particles with some inner structure. On observing Figs. 5(a) and 5(b), one can notice how the clusters are all the branches or trees, which stem from the main body of the aggregates. In Fig. 5(e), the solid line represents the DLA packing factor $\kappa$ as a function of $\zeta$; in the same diagram, the points in light green describe the packing degree of the experimental clusters. The theoretical model recovers the experimental results with appreciable accuracy, and both confirm that, while the packing factor increases with the pattern distance, this effect gradually reduces with $\zeta$. When $\zeta$ is sufficiently large, the electroless silver deposition would not depend on the pattern distance. 

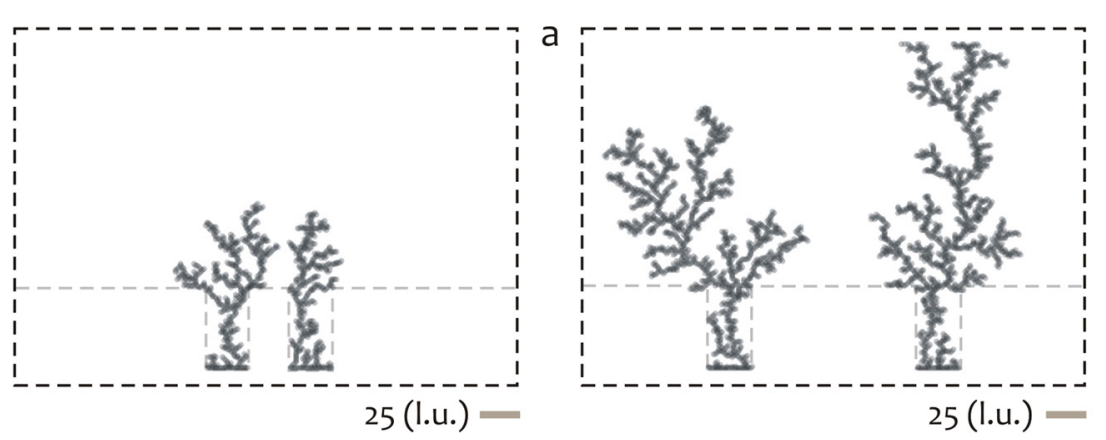

d

\begin{tabular}{|c|c|c|c|c|}
\hline $\begin{array}{c}\text { Distance } \\
(\zeta)\end{array}$ & $\begin{array}{c}\text { SEM Particle } \\
\text { Size }(\mathrm{nm})\end{array}$ & $\begin{array}{c}\text { DLA Particle } \\
\text { Size (I.u.) }\end{array}$ & $\begin{array}{c}\text { SEM Packing } \\
\text { Factor }\end{array}$ & $\begin{array}{c}\text { DLA Packing } \\
\text { Factor }\end{array}$ \\
\hline 1 & $82 \pm 4$ & $27 \pm 3$ & 85 & 81 \\
\hline 1.5 & $110 \pm 6$ & $36 \pm 5$ & 108 & 108 \\
\hline 2 & $130 \pm 11$ & $45 \pm 4$ & 132 & 135 \\
\hline 3 & $128 \pm 10$ & $42 \pm 5$ & 129 & 126 \\
\hline 4 & $148 \pm 9$ & $52 \pm 6$ & 153 & 156 \\
\hline
\end{tabular}

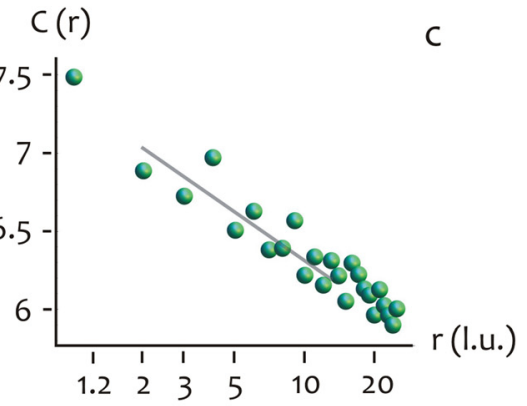

$\kappa(\mathrm{E}-4)$

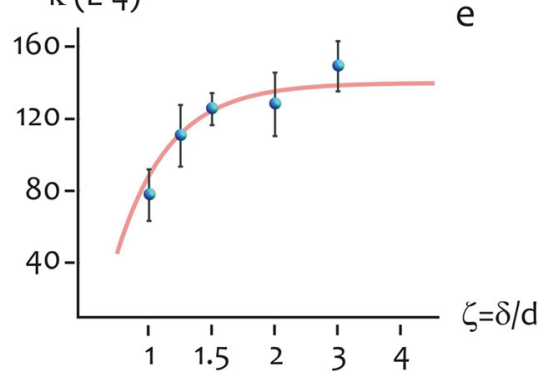

FIG. 5. (Color online) (a) DLA aggregates obtained for $\zeta=1$. (b) DLA aggregates obtained for $\zeta=4$. (c) Power spectrum density function of numerical aggregates. From the diagram, the fractal dimension $D f$ is derived, being $D f=5 / 3$. (d) The table recapitulates the values of mean cluster size $\mathrm{S}$ and packing factor $\kappa$, derived from the experimental SEM images of the aggregates, and predicted by the DLA simulations. (e) The experimental (points in blue) and numerical (solid line) packing factor $\kappa$ as a function of the ratio of the distance to the pattern diameter, $\zeta=\delta / d$.

In the presented analysis, we used a distance normalized to the diameter of the pattern and this would set us free from a specific pattern size. In other words, the diagram presented in Fig. 5(e) is a very general result that predicts that for, a generic pattern size, the packing factor (that is somehow correlated to the characteristic length scale) of a structure may vary from 1 to 3 times its initial value, on changing the pattern distance from 1 to 3 times the pattern diameter. This understanding may be combined with the effect of pattern size obtained in Ref. 14 to yield a complete description of electroless deposition as a function of the geometry of the substrate.

\section{Discussion}

The discovery of fundamental principles of how nanoscale systems of building blocks assemble enables to engineer new nanomaterials with enhanced properties compared to their macroscopic counterparts. ${ }^{32-34}$ This would be the case of metal nanomaterials, toward which there is a mounting interest for a variety of factors that include, but are not restricted to, an uncommon ability of interacting with light, ${ }^{35}$ small sizes, at which much of biology occurs, ${ }^{2}$ a high degree of biocompatibility ${ }^{36,37}$ not complicated procedures for biofunctionalization $^{38,39}$ and elevated surface to volume ratios. ${ }^{40,41}$ Some of the cited properties may constitute the ground for new families of advanced devices, and especially SERS devices, with augmented functions for applications in biology and biotechnology. ${ }^{42,43}$

In Ref. 11, we proposed an integrated bottom-up-topdown approach to produce ordered arrays of silver optical nanolenses. In the method, silver nanoparticles aggregates are deposited (that is, the bottom up part of the process, 1) within well-defined patterns created by electron beam lithography on silicon substrates (that is, the top down part of the process, 2) where electroless deposition ${ }^{4-46}$ is a chemical process based upon reduction from metallic ions and allows precise control over the nucleation and growth of nanoparticles.

In Ref. 47, experiments and exploratory molecular dynamics simulations of self-aggregation in cylindrical nanostructures permitted to decipher the rules governing the evolution of the silver nanoparticles growth front in devices where the aspect ratio was varied over a significant range. In Ref. 14, a DLA simulation method was utilized to gain insight into the mechanisms of electroless formation in aggregates in which the size of the patterns is varied over a significant range. The cited last two examples support the notion that pioneering nanofabrication techniques should be accompanied by appropriate theoretical models.

Here, we expanded and improved the DLA model utilized in Ref. 14 to address and resolve the multi-scale problem of electroless particle formation as a function of pattern distance. The use of a DLA framework is motivated by mathematical convenience and the accuracy of the model to explore ways to tailor film morphology to obtain specific characteristics. This scheme was utilized to predict the electroless growth of nonperiodic geometries; in doing this, we demonstrated that the spacing of the patterns of deposition is an important factor that should be correctly considered in the design of devices where particles' aggregation and the derived effects thereof play an important role.

In Fig. 6, additional experiments confirm the predictions of the DLA model. Particles were realized with a nominal 


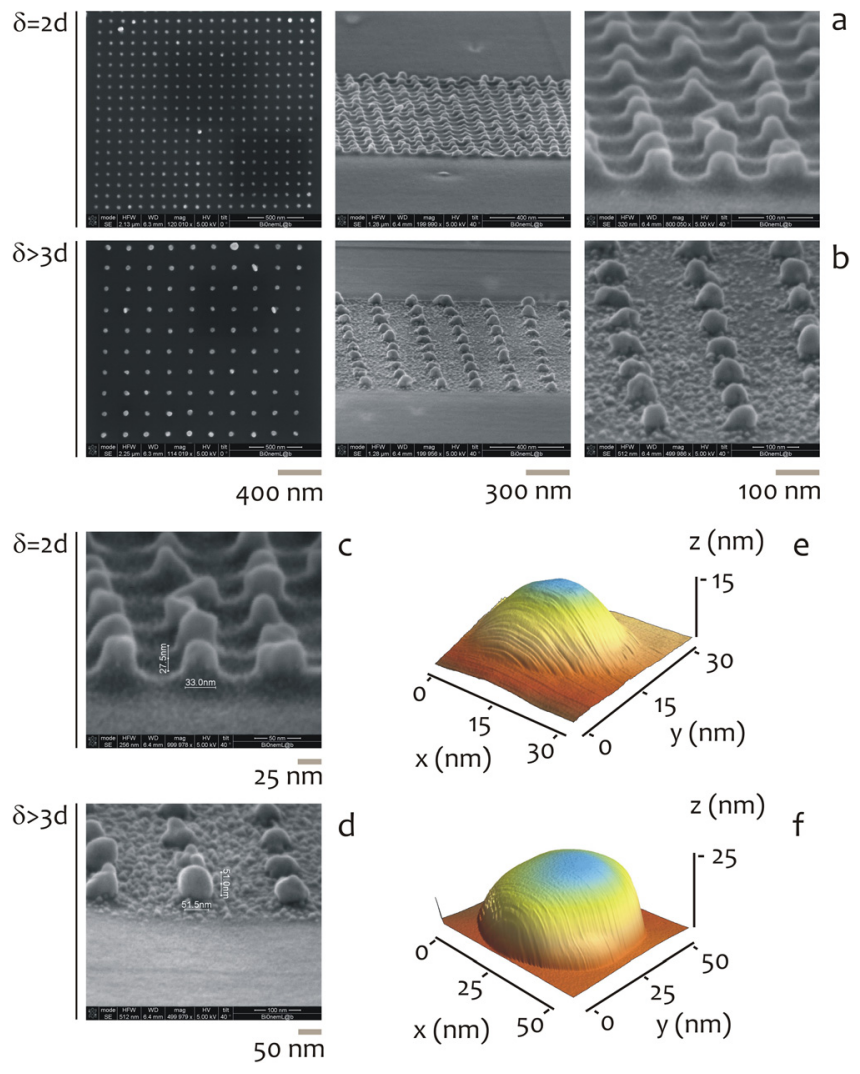

FIG. 6. (Color online) (a) SEM micrograph of a lattice of silver nanoparticles, where the pattern distance $\delta=2 d$ is small, thus influencing the effective size of the nanoparticles that, for the present configuration, is $d=30 \mathrm{~nm}$. In (b), SEM image reveals that in a lattice where the pattern distance is sufficiently large $(\delta>3 d)$, the effective size of the nanoparticles is larger, being $d=50 \mathrm{~nm}$. (c) Ultrahigh resolution image of a silver particle in a small packing pattern $(\delta=2 d)$; for this configuration, the particle reveals an oblate shape with an effective size of $30 \mathrm{~nm}$ that is roughly half the size of the nominal diameter. (d) Ultrahigh resolution image of a silver particle in a large packing pattern $(\delta>3 d)$; for this configuration, the particle reveals a spherical shape with an effective size of $50 \mathrm{~nm}$ that is practically equal the nominal diameter. (e) AFM profile of a nanoparticle in a pattern with a small lattice constant $(\delta=2 d)$. (f) AFM profile of a nanoparticle in pattern with a large lattice constant $(\delta>3 d)$

size of $d=50 \mathrm{~nm}$ and a varying particle distance. When the particles are in close proximity, as in Fig. 6(a) $(\delta=2 d)$, their size or dimension is reduced; differently from this, when the particles are separated as in Fig. 6(b) $(\delta>3 d)$, the dimension of the spheres is augmented with no other constraints. Remarkably, the variation in size is consistent with the diagram in Fig. 5(e). These findings are supported by the ultrahigh resolution SEM micrographs in Figs. 6(c) and 6(d) and by the AFM images in Figs. 6(e) and 6(f), where the particles in a large packing pattern [Figs. 6(d) and 6(f)] exhibit a neat spherical shape with an effective diameter of about $50 \mathrm{~nm}$ that differs slightly from the nominal size; and particles in a small packing pattern [Figs. 6(c) and 6(e)] reveal an elongated, oblate shape with an effective diameter of about $30 \mathrm{~nm}$, which is roughly the half of the nominal size.

This original information should be used for the correct design of metal nanopatterns. The packing factor presented in Fig. 5(e) has the meaning of the effective particle diameter for a given pattern spacing. The size increment, or gain, with respect to a lattice where the spacing between the patterns equals the diameter of those patterns $(\delta=d)$ is a function of the pattern distance $\delta$. Let us call this increment $\eta(\delta)$ $=\kappa(\delta) / \kappa(1)$. Therefore, $\eta(\delta)$ can be regarded as a multiplication factor by which a nominal pattern of size $d_{0}$ has to be multiplied to obtain an effective pattern of size $d=\eta d_{0}$, in an array of structures where the lattice constant is $\delta$.

This understanding may be combined with the effect of pattern size derived in Ref. 14 to obtain design maps for electroless metal nanomaterials. These design maps (currently under development, and of which in Fig. 8 in Appendix $\mathrm{C}$ is reported a preliminary representation), derived for specific values of time and temperature of deposition, would give a quantitative indication of the growth efficiency (that is, $\eta$ ) of a system as a function of diameter and gap of the pattern. Their usage would be simple. An engineer interested in producing a particular metal nanopattern can enter the diagram with specific values of (nominal) size and gap and read the effective diameter increment that the structure would reveal for fixed conditions of growth.

Somewhat similar in concept to the periodic table of chemical elements, but more akin to phase diagrams in their graphic presentation, these design maps may provide guidance for the development of optimized metal nanomaterials obtained via electroless deposition for a variety of applications. These maps and their more sophisticated evolution that will be developed over time will allow for the fabrication of metal, electroless grown nanomaterials on a quantitative basis, similarly to the design of experiments concept routinely utilized in engineering.

\section{SUMMARY AND CONCLUSIONS}

Silver NPs aggregates with an overall size in the nanometer range were fabricated employing nonconventional site selective electroless techniques and electron beam lithography. Electroless is a novel deposition technique in which metal ions are reduced as atoms on specific patterned sites of a silicon surface, to form nanoparticulates with the desired characteristics. In varying the spacing of the patterns over a significant range, we observed a very high sensitivity of the roughness of the resulting aggregates to the distance of the patterns. More specifically, we found that the efficiency of growth increases with the distance, in the sense that when the clusters are closer, the packing factor of these is low and, vice versa, when the patterns are sufficiently distant, the packing factor is large. Here, close and distant have the particular meaning of smaller and larger than a particular threshold, that is, a correlation distance below which the interference between the clusters is significant. Therefore, a number of clusters that are farther from each other than this correlation distance would behave like a set of independent systems and the electroless growth, the NPs aggregates and the properties thereof can be described like those of an isolated system. This critical limit, for the particular subset of parameters used here, is about three times the pattern diameter.

These experimental findings were explained within the framework of a DLA model where it is assumed that, in the 
limit of very fast chemical reactions, diffusion is the sole driving force that regulates the dynamics of aggregation of NPs. Collectively, these results suggest that the distance between different groups of NPs clusters is an important parameter that cannot be neglected in designing devices that utilize rough metal surfaces and their effects, including the very large area of SERS substrates, especially pertaining the single molecule detection issue.

\section{NOMENCLATURE}

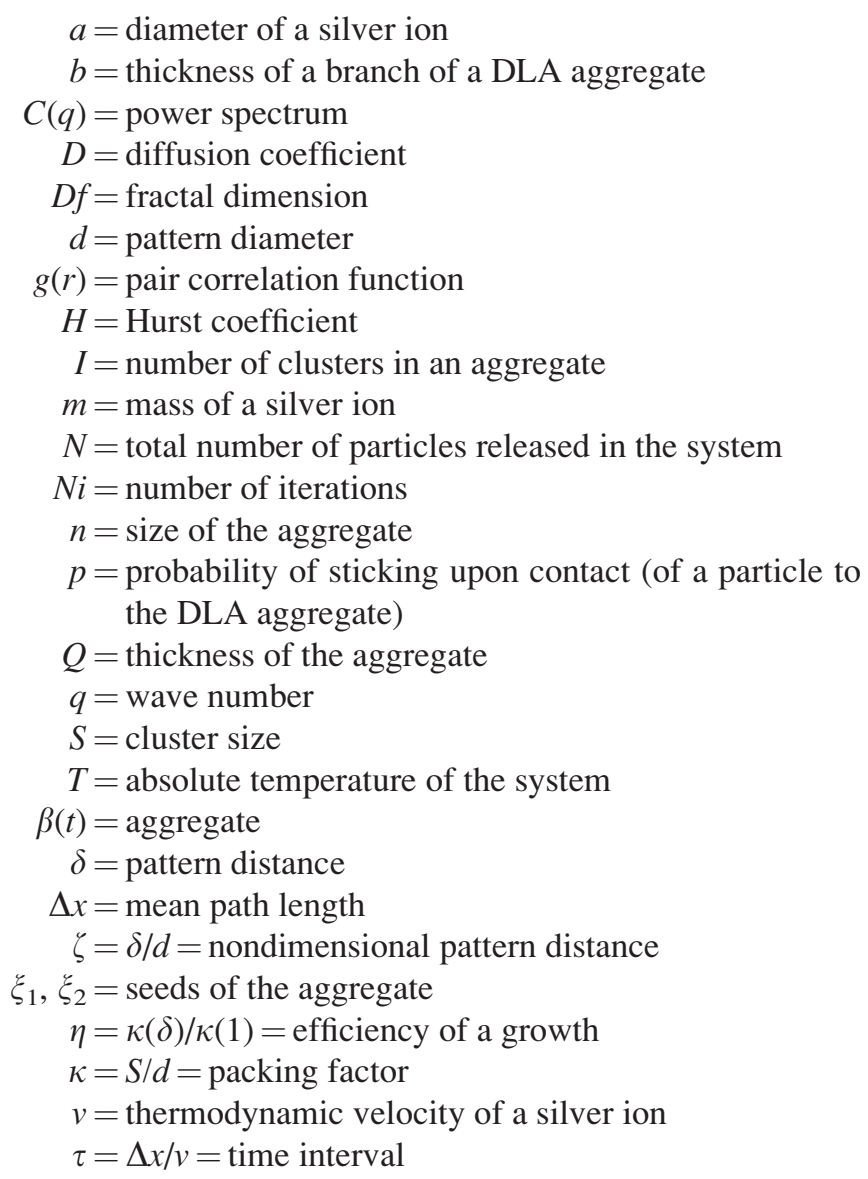

\section{ACKNOWLEDGMENTS}

This work has been partially funded from the EU Commission, the European Social Fund, and the Calabria Region (POR Calabria FSE 2007-2013) and from the Italian Minister of Health (Project No. GR-2010-2320665).

\section{APPENDIX A}

In Fig. 7, we schematically show the local concentrations effects, that is, to which extent metal ions are available to growth in close proximity of a pattern. Every pattern (well) would have a certain number $N$ of metal ions immediately above and beside it, comprised in certain region of interest. These particles represent the candidates for being deposited in a single pattern in a given amount of time. When the patterns are sufficiently close, the cited regions of interest would to a certain extent superimpose, and thus, the free particles available for growth would reduce per each pattern.
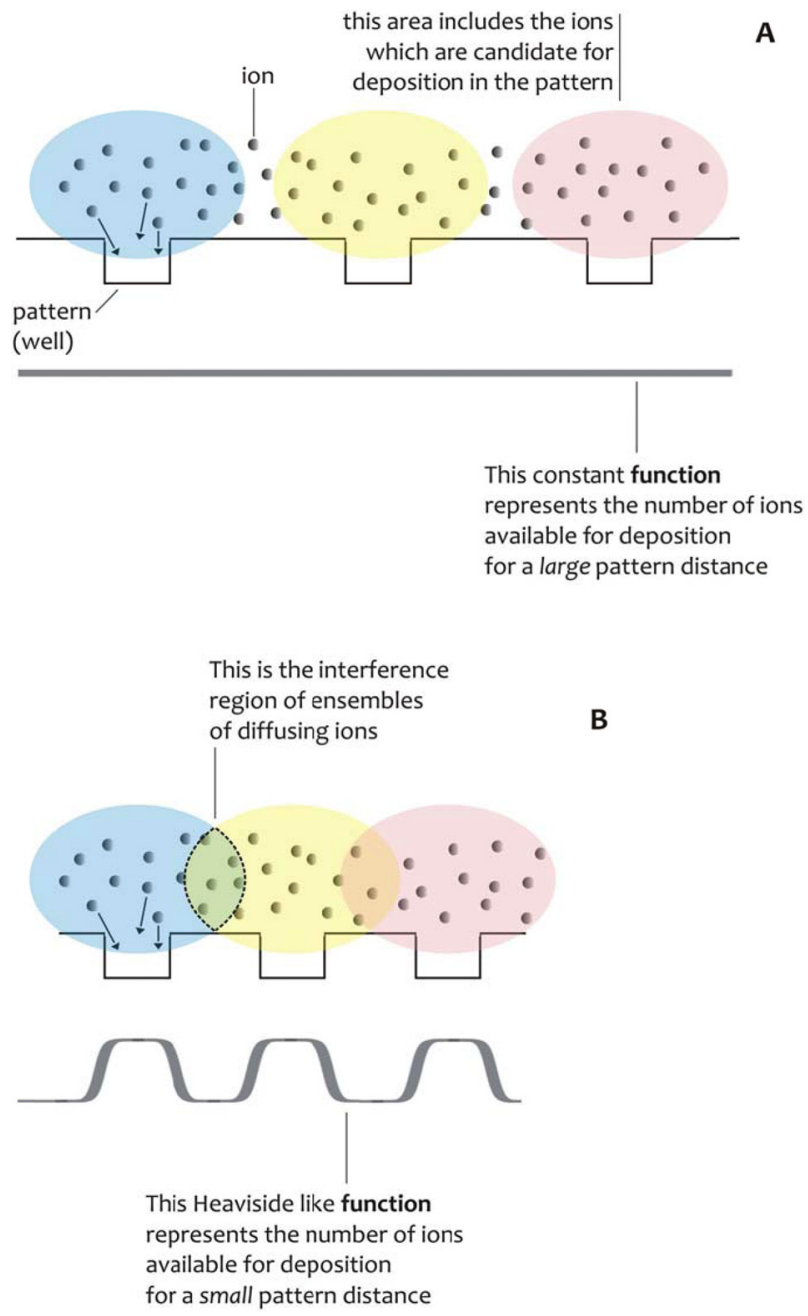

FIG. 7. (Color online) Figure represents the number of ions which are candidate for being deposited into the pattern, for a large (A) and small (B) pattern distance.

\section{APPENDIX B}

On observing the clusters of silver nanoparticles deposited on different circular patterned surfaces, one can notice that the growth is more pronounced on the borders or edges of those patterns. This effect can be ascribed to a nonuniform distribution of surface energy density, which, on the edges, is larger than on flat surfaces.

Nanostructures and nanomaterials possess a large fraction of surface atoms per unit volume. The ratio of surface atoms to interior atoms changes dramatically if one successively divides a macroscopic object into smaller parts. For example, for a cube of iron of $1 \mathrm{~cm}^{3}$, the percentage of surface atoms would be only $10^{-5} \%$. When the cube is divided into smaller cubes with an edge of $10 \mathrm{~nm}$, the percentage of the surface atoms would increase to $10 \%$. This mechanism is responsible for the augmentation of surface energy of at the edges or perimeter of a surface. While the surface energy of flat silicon is $\gamma=1.82 \mathrm{~J} / \mathrm{m}^{2}, 49$ at the edges of silicon $\gamma$ can be larger of several orders of magnitude. In those areas, the materials are highly reactive and would induce a faster deposition, reduction, and formation of silver atoms into aggregates. 


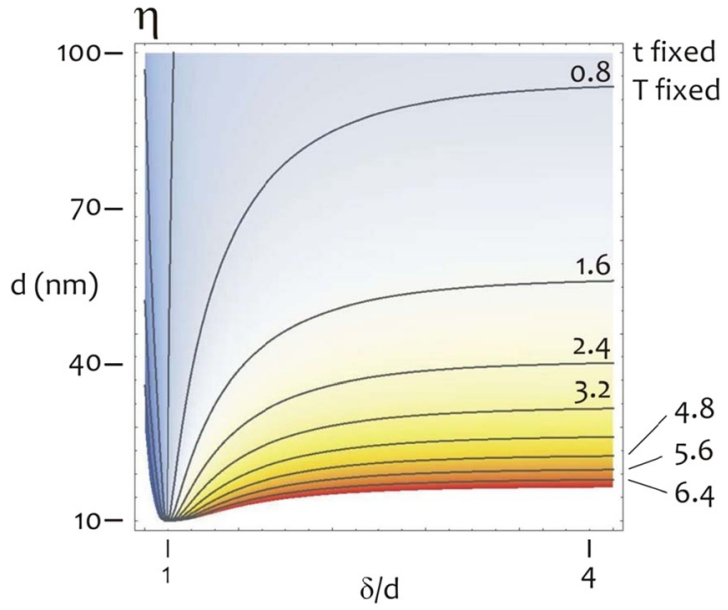

FIG. 8. (Color online) Example of design map, where the efficiency of particle growth is reported in the form of a contour plot as a function of pattern size and pattern distance.

The classical nucleation theory predicts the critical nucleation diameter in homogeneous nucleation as

$$
\begin{aligned}
& \mathrm{dc}=-4 \gamma / \Delta \mathrm{Gv}, \\
& \Delta \mathrm{Gv}=-\mathrm{K}_{\mathrm{b}} \mathrm{T} /\left(\Omega \ln \left(\mathrm{C} / \mathrm{C}_{0}\right)\right),
\end{aligned}
$$

where $\Delta \mathrm{G}_{\mathrm{v}}$ the change of Gibbs free energy per unit volume of the solid phase, $\Omega=1.1610^{-29} \mathrm{~m}^{3}$ is the volume of a silver atom, $\mathrm{C} / \mathrm{C}_{0}=10$ is the super saturation of the actual to the equilibrium concentration of the system, $\mathrm{K}_{\mathrm{b}}$ is the Boltzmann constant, and $\mathrm{T}=323 \mathrm{~K}$ is the temperature of the system expressed in Kelvin degrees. Equation (B1) is the result of a simple balance between surface energy and Gibbs free energy per unit volume. The probability of a thermodynamic fluctuation associated to $\gamma$ is

$$
\mathrm{p}_{0}=\mathrm{e}^{-16 \pi \gamma /(3 \mathrm{kT} \Delta \mathrm{Gv} 2)},
$$

and, given that $\Delta \mathrm{G}_{\mathrm{v}}$ is negative, Eq. (B2) claims that larger $\gamma$ would favor the formation and growth of nanograins, in agreement with the experimental results.

\section{APPENDIX C}

Here, we show an example of how a design map for electroless metal nanomaterials would look like. These design maps, derived for specific values of time and temperature of deposition, would give a quantitative indication of the growth efficiency (that is, $\eta$ ) of a system as a function of diameter and gap of the pattern. Their usage would be simple. An engineer interested in producing a particular metal nanopattern can enter the diagram with specific values of (nominal) size and gap, and read the effective diameter increment that the structure would reveal for fixed conditions of growth.

${ }^{1}$ T. Theis et al., Nat. Nanotechnol. 1, 8 (2006).

${ }^{2}$ G. Whitesides, Nat. Biotechnol. 21, 1161 (2003).
${ }^{3}$ M. Ferrari, Nat. Rev. Cancer 5, 161 (2005).

${ }^{4}$ G. Das, F. Mecarini, F. Gentile, P. Candeloro, C. Liberale, F. De Angelis, H. Kumar, G. Cuda, and E. Di Fabrizio, Biosens. Bioelectron. 24, 1693 (2009).

${ }^{5}$ K. Kneipp, Phys. Today 60(6), 40 (2007).

${ }^{6}$ F. Garcia-Vidal and J. Pendry, Phys. Rev. Lett. 77, 1163 (1996).

${ }^{7}$ C. L. Phillips, J. A. Anderson, G. Huber, and S. C. Glotzer, Phys. Rev. Lett. 108, 198304 (2012).

${ }^{8}$ P. F. Damasceno, M. Engel, and S. C. Glotzer, Science 337, 453 (2012).

${ }^{9}$ S. Glotzer, Nature 481, 450 (2012).

${ }^{10}$ Y. Xia, T. D. Nguyen, M. Yang, B. Lee, A. Santos, P. Podsiadlo, Z. Tang, S. C. Glotzer, and N. A. Kotov, Nat. Nanotechnol. 6, 580 (2011).

${ }^{11}$ M. L. Coluccio et al., Microelectron. Eng. 86, 1085 (2009).

${ }^{12}$ T. Qiu and P. Chu, Mater. Sci. Eng. R 61, 59 (2008).

${ }^{13}$ A. R. Tao, S. Habas, and P. Yang, Small 4, 310 (2008).

${ }^{14}$ F. Gentile et al., Microelectron. Eng. 98, 359 (2012).

${ }^{15}$ T. Qiu, X. Wu, Y. Mei, P. Chu, and G. ùSiu, Appl. Phys. A 81, 669 (2005).

${ }^{16}$ T. Witten and L. Sander, Phys. Rev. Lett. 47, 1400 (1981).

${ }^{17}$ R. Dawkins and D. ben-Avraham, Comput. Sci. Eng. 3, 72 (2001).

${ }^{18} \mathrm{H}$. Wu, M. Lattuada, P. Sandkuhler, J. Sefcik, and M. Morbidelli, Langmuir 19, 10710 (2003).

${ }^{19}$ F. Persson, P. Utko, W. Reisner, N. Larsen, and A. Kristensen, Nano Lett. 9, 1382 (2009).

${ }^{20}$ Z. Zhang and M. Lagally, Science 276, 377 (1997).

${ }^{21}$ A. R. Howells, L. Hung, G. S. Chottiner, and D. A. Scherson, Solid State Ionics 150, 53 (2002).

${ }^{22}$ T. Qiu, X. Wu, G. Siu, and P. K. Chu, Appl. Phys. Lett. 87, 223115 (2005).

${ }^{23}$ A. Kuhn and F. Argoul, J. Electroanal. Chem. 397, 93 (1995).

${ }^{24}$ Z. Liu, H. Lee, Y. Xiong, C. Sun, and X. Zhang, Science 315, 1686 (2007).

${ }^{25}$ M. Saltzmann, Drug Delivery (Oxford Univ. Press, Oxford, UK, 2001).

${ }^{26}$ P. Decuzzi, F. Gentile, A. Granaldi, A. Curcio, F. Causa, C. Indolfi, P. Netti, and M. Ferrari, Int. J. Nanomed. 2, 689 (2007), available at http:// www.dovepress.com/articles.php?article_id=712.

${ }^{27}$ F. Gentile, L. Tirinato, E. Battista, F. Causa, C. Liberale, E. Di Fabrizio, and P. Decuzzi, Biomaterials 31, 7205 (2010).

${ }^{28}$ P. Meakin, Phys. Rev. B 30, 4207 (1984).

${ }^{29}$ Z. Racz and T. Vicsek, Phys. Rev. Lett. 51, 2382 (1983).

${ }^{30}$ F. Gentile et al., Microelectron. Eng. 88, 2537 (2011).

${ }^{31}$ F. Gentile et al., Microelectron. Eng. 111, 272 (2013).

${ }^{32}$ A. Carpentieri and N. Pugno, Nat. Mater. 4, 421 (2005).

${ }^{33}$ Y. Astier, L. Data, R. P. Carney, F. Stellacci, F. Gentile, and E. Di Fabrizio, Small 7, 455 (2011).

${ }^{34}$ M. Ferrari, Small 4, 20 (2008).

${ }^{35}$ K. L. Kelly, E. Coronado, L. L. Zhao, and G. C. Schatz, J. Phys. Chem. B 107, 668 (2003).

${ }^{36}$ K. Lee, P. Nallathamby, L. Browning, C. Osgood, and X. -H. N. Xu, ACS Nano 1, 133 (2007).

${ }^{37}$ R. Bhattacharya and P. Mukherjee, Adv. Drug Deliv. Rev. 60, 1289 (2008).

${ }^{38}$ A. Ravindrana, A. Singha, A. M. Raichurb, N. Chandrasekarana, and A. Mukherjeea, Colloid Surf. B 76, 32 (2010).

${ }^{39}$ C. M. Niemeyer, Angew. Chem. Int. Ed. 40, 4128 (2001).

${ }^{40}$ E. Roduner, Chem. Soc. Rev. 35, 583 (2006).

${ }^{41}$ S. Cuenot, C. Frétigny, S. Demoustier-Champagne, and B. Nysten, Phys. Rev. B 69, 165410 (2004).

${ }^{42}$ F. De Angelis et al., Nat. Photon. 5, 682 (2011).

${ }^{43}$ F. Gentile et al., ACS Appl. Mater. Interfaces 4, 3213 (2012).

${ }^{44}$ H. Natter and R. Hempelmann, Electrochim. Acta 49, 51 (2003).

${ }^{45}$ C. R. K. Rao and D. C. Trivedi, Coordin. Chem. Rev. 249, 613 (2005).

${ }^{46}$ M. Schlesinger and M. Paunovic, Modern Electroplating (Wiley, New York, 2000).

${ }^{47}$ W. Babiaczyk, S. Bonella, G. Ciccotti, M. Coluccio, F. Gentile, and E. Di Fabrizio, Nanoscale 4, 2362 (2012).

${ }^{48}$ W. M. Haynes, CRC Handbook of Chemistry and Physics (CRC, Boulder, CO, 1998).

${ }^{49}$ A. A. Stekolnikov, J. Furthmuller, and F. Bechstedt, Phys. Rev. B 65, 115318 (2002). 